\title{
UNA PROPUESTA DE MEJORAMIENTO DEL TRANSPORTE PÚBLICO COLECTIVO EN SANTIAGO DE CALI
}

Rolf Moller*

* Sociólogo, Universidad de Biecefeld, Alemania.

Profesor Asistente, Área de Gestión Ambiental, Escuela de Ingeniería de Recursos Naturales y del Ambiente Facultad de Ingeniería - Universidad del Valle.

\section{RESUMEN}

Se presentan las deficiencias del sistema de transporte público colectivo actual en Santiago de Cali, en especial los efectos negativos de la llamada 'guerra del centavo', para los pasajeros, los conductores, las empresas prestadoras del servicio de transporte urbano, los ciudadanos y la economía local. Se explican las deficiencias y problemas actuales como consecuencia lógica de la 'guerra del centavo' y de sus elementos estructurales, para finalmente presentar una propuesta para la creación de un sistema de transporte público colectivo más eficiente, agradable, rápido, barato y ambiental, social y 
económicamente sostenible. Se trata de mostrar que sería posible mejorar el transporte público colectivo en la ciudad significativamente a través de una profunda reorganización del actual sistema de transporte con buses, sin necesidad de una costosa inversión en infraestructura vial o en tecnologías como el metro. Se trata de mostrar cómo esta reorganización mejora la situación ambiental, crea condiciones de equidad social, puede contribuir a la disminución de la pobreza y al aumento de la competitividad de las empresas en Santiago de Cali.

\section{ABSTRACT}

This article presents the deficiencies of the current system of public transportation in Cali, especially the negative effects of the so called "war for the cents." This system affects greatly passengers, bus drivers, transportation companies, citizens, and also the local economy. The deficiencies are explained as a logical consequence of the "war for the cents" and all its structural elements. Finally, a proposal is presented for the creation of a more efficient, agreeable, quicker, cheaper, and for an environmentally, socially, and economically sustainable public transportation system. The purpose is to demonstrate that it is possible to improve substantially the public transportation system through a profound reorganization of public transportation system, without an expensive investment in technology of a subway or light rail train. We intent to demonstrate that this reorganization will significantly improve the environmental situation, create conditions of social equity, contribute to reduce poverty and in one way improve the efficiency of the bus companies in Santiago de Cali.

\section{EL DIAGNÓSTICO DE LAS CONDICIONES Y DE LA SITUACIÓN ACTUAL EN EL TRANSPORTE PÚBLICO COLECTIVO EN CALI}

El punto de partida para cualquier solución del transporte público colectivo en Cali siempre debe ser el diagnóstico detallado de la actual situación.

A primera vista el sistema de transporte público colectivo en Cali tiene sus méritos: según un estudio realizado por la Secretaría de Tránsito $^{1}$ cada día se realizan cerca de 1.4 millones de viajes $^{2}$ en los cerca de 5.000 buses, busetas, microbuses y camperos 3 de las 29 empresas $^{4}$ que realizan la movilización de personas. "Para el $63 \%$ de los usuarios de buses, el servicio ...es bueno, pero hay un $37 \%$ que cree que ese servicio es malo."

Un diagnóstico detallado muestra las deficiencias, a veces no tan obvias, del actual sistema de transporte público colectivo en buses. Para un diagnóstico más detallado y profundo hay que tener en cuenta la situación y los respectivos intereses de todas las personas que están involucrados en, o afectados por el sistema de

${ }^{1}$ El estudio "Diagnóstico del Sistema de Transporte Público Colectivo de la Ciudad de Santiago de Cali" realizado por la Secretaría de Tránsito y Transporte Municipal" data de junio de 2000.

2 El estudio habla de 1.282.201 por día. Ibíd. P. 22. Esta cifra no incluye los pasajeros que los conductores permiten viajar sin pago, y los pasajeros que suben por la puerta atrás sin ser registrados. Se estima el número de pasajeros no contados en cerca de 150.000 por día, con base en observaciones personales.

3 Ibíd. P. 8

${ }^{4}$ Ibíd. P. 8

5 MELO GARCÍA, Luyith. Los pecados del transporte público. El País, p. 1B, mayo 14 de 2000. 
transporte público colectivo de la ciudad directa e indirectamente. En especial hay que ver la situación de los pasajeros actuales y posibles usuarios futuros, de los conductores, de las empresas prestadoras del servicio y sus afiliados, de los propietarios de buses, de la ciudadanía, de las instituciones estatales como la Secretaría de Tránsito y Transporte, del sector empresarial, de los otros participantes en el transporte urbano como los peatones, ciclistas y los conductores de vehículos motorizados.

\section{LAS DEFICIENCIAS DEL TRANSPORTE PÚBLICO COLECTIVO EN CALI, DESDE EL PUNTO DE VISTA DE SUS DIFERENTES ACTORES}

\section{El transporte público colectivo desde el punto de vista del pasajero.}

El sistema de transporte en buses actualmente tiene una serie de deficiencias desde el punto de vista del pasajero.

Los buses son lentos porque paran constantemente para recoger y dejar pasajeros, se frenan mutuamente. Son a la vez causa y víctima de los trancones, dan largas vueltas en los barrios.

Los buses no son calculables ni confiables. El pasajero no puede saber a qué hora va a pasar el bus, menos aún los domingos, festivos y en horas de la noche o muy temprano. El pasajero no

\footnotetext{
6 Algunas de las críticas se encuentran en el articulo de Melo García, Ibíd. La lista completa es el resultado de observaciones personales, confirmadas en cursos sobre el tema del transporte urbano por cientos de estudiantes de la Universidad del Valle que regularmente son usuarios de los buses.

7 La velocidad promedio es de $20 \mathrm{~km}$. / hora, con la empresa más lenta con un promedio de 14.1 y la más rápida con $27.1 \mathrm{~km}$. / hora. Ver Estudio de la Secretaría de Tránsito, Ibíd. p. 22
}

puede saber cuándo va a tener una conexión ni cuánto tiempo durará su recorrido.

Hay un mal o ningún servicio en horas de la noche. Los domingos y festivos se reduce significativamente la oferta. En la noche después de las 9 p.m. hay escaso servicio más tarde no hay buses.

En partes de la ciudad, en especial en el centro, hay una sobreoferta de buses que se frenan mutuamente. En otras partes de la ciudad, en especial en los barrios marginados no hay o sólo hay un mal servicio.

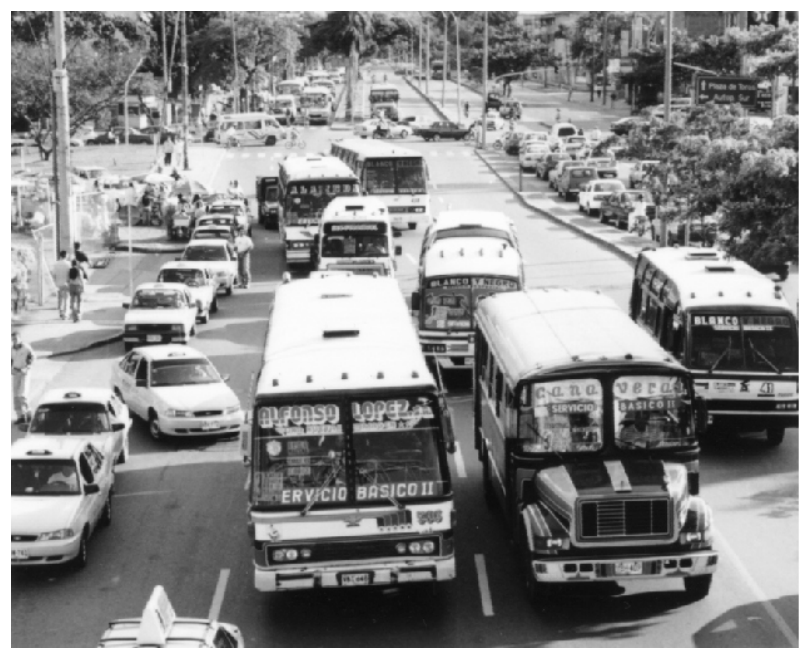

La sobreoferta de buses en diferentes partes de la ciudad. -Aquí en la Calle 5, frente al Éxitohace que los buses se frenen mutuamente. Entre otros, por eso el transporte público colectivo en Cali es tan lento.

Muchos buses son viejos, a veces sucios, algunos poco cómodos, por ejemplo los camperos.

Especialmente los microbuses tienen sillas poco cómodas, muy estrechas. Personas de baja estatura tienen dificultades para llegar al timbre.

8 Según MELO GARCÍA los buses tienen en promedio entre 8 y 14 años de vida, el $20 \%$ más que 20 años. Ibíd. 
Muchos conductores están acostumbrados a conducir agresivamente, aceleran y frenan bruscamente, realizan maniobras peligrosas para recoger y dejar pasajeros.

El ruido de la música y del motor dentro del bus molesta a los pasajeros.

Las gradas y las registradoras dificultan la subida y bajada para niños, para personas de baja estatura, para personas con limitaciones físicas, para personas con paquetes, para gordos y para padres con niños en los brazos.

Los pasajeros están expuestos a la contaminación, desde dentro y fuera del bus. Mo sólo sufren de la contaminación atmosférica que entra desde fuera del bus, a la cual los buses contribuyen una parte significativa -, sino desde dentro del bus: a veces el motor no está bien encapsulado y entran los gases de la combustión.

Los pasajeros corren riesgos de accidentes subiendo y bajando, por paradas en la mitad de la calle, y pueden sufrir lesiones o golpes porque los conductores arrancan mientras el pasajero sube o baja las gradas y cuando está de pie y el bus frena.

Los pasajeros pueden ser víctimas de atracos, asesinatos, violaciones, especialmente en la noche y en barrios marginados.

El actual sistema de transporte público colectivo produce exclusión social y económica. Los buses no permiten la movilidad de personas con limitaciones físicas (silla de ruedas, ciegos, limitaciones para subir gradas etc). El precio del viaje no permite el uso de los buses para todos los que quieren movilizarse, en especial en familias de estratos bajos. Los buses no llegan a todos los sitios (culturales) de la ciudad, en Cali por ejemplo es difícil llegar al Museo La Tertulia. En barrios marginados y en la noche no hay servicio: los que regresan después del juego de fútbol o de un concierto tienen que pagar taxi o ir a pie.

Quienes no conocen muy bien la ciudad (viajeros, extranjeros, y los mismos ciudadanos) no saben qué ruta tomar para llegar a cierto punto de la ciudad. Existe una completa ausencia de información en los paraderos.

Finalmente se presentan muchos conflictos y peleas entre pasajeros y el conductor, por ejemplo cuando éste espera para recoger más pasajeros y hace esperar a todos, cuando no puede dar la vuelta o se 'equivoca', cuando viaja con música muy fuerte o cuando no para donde el pasajero lo espera, etc.

\section{El transporte público colectivo desde el punto de vista del conductor:}

También los conductores sufren mucho en la actual organización del transporte público colectivo en Cali, por ejemplo a través de largas horas laborales. El día laboral de muchos de los conductores se extiende a $12^{9}$ y más horas laborales por día, por las largas distancias de los recorridos, para ganar más o hacer viable la inversión del bus, por obligación por parte del propietario del bus, con efectos nocivos para la salud, los contactos sociales y la vida familiar del conductor.

A pesar de las largas horas de trabajo los ingresos de los conductores son muy modestos. Dependiendo de si el conductor es propietario del bus, propietario en sociedad o conductor 'contratado', los salarios varían entre uno y cuatro salarios mínimos. De los conductores contratados el19,2\% ganan hasta un salario mínimo, el $44,7 \%$ entre uno y dos salarios mínimos, el 25.8 entre dos y tres, el $9.3 \%$ entre tres y cuatro, y el $1 \%$ más de cuatro salarios mínimos.

\footnotetext{
9 Para Cali son un promedio de 11,9 horas, según el Ministerio de Salud, Centro Macional de Consultoría, en el año 1995. Sólo $7.9 \%$ de los conductores trabajan 8 horas o menos, 29,4\% entre 9 y 12 horas, $42.1 \%$ entre 13 y 16 horas y todavía $20.6 \% 17$ y más horas por día. Corporación Salud y Desarrollo. El Bienestar del Conductor del Transporte Público Urbano. Seguro Social Protección Laboral, Bogotá (sin año) 1998 ?), p. 33,75 . Por la crisis económica, que vive el país desde mitad del año 1998, puede ser que las horas laborales de los conductores han aumentado aún más.
} 
Los ingresos de los propietarios son algo mejores, pero inclusive entre ellos sólo cerca del 10\% ganan más de cuatro salarios mínimos. El 64.7\% tienen que sostener entre 3 y 5 personas a su cargo, el $8.1 \%$ a seis y más personas. ${ }^{10}$ Es obvio que los ingresos en muchos casos están cerca o por debajo de la línea de pobreza.

Las presiones de buscar pasajeros a toda costa resultan en altos riesgos de accidentes con otros buses, carros, motos, de provocar una caída de pasajeros dentro del bus, de matar a un peatón en la calle, de caerse en un canal o de chocar con un poste de energía eléctrica. En Cali, un 37.3\% de los conductores informaron haber tenido un accidente de tránsito en los últimos 6 meses?

Los conductores están constantemente bajo presión y estrés, para ganarse la vida con suficientes pasajeros, realizando múltiples funciones a la vez: cobrar, mirar los espejos, observar el tráfico adelante, observar la competencia, frenar y acelerar, darse cuenta de las señales de los pasajeros, dar información, etc. El estrés ocupa uno de los primeros puestos entre las molestias de salud relacionadas con el trabajo, expresadas por los conductores en 1996: 19.7\% mencionan el estrés.2. Manejar un bus significa altos riesgos para la salud $y$ enfermedades. ${ }^{13}$

10 lbíd., P. 91/95. El estudio presenta un análisis de la situación de conductores en Bogotá, Cali y Medellín. Mo se presentan datos específicos para Cali sobre los ingresos y las personas a cargo.

11 Ibíd. P.47

12 Ibíd. P. 41

13 En el estudio aparecen una larga lista de enfermedades y de síntomas ocupacionales informados por los conductores en el anamnesis clínica en Bogotá: dolor de cintura, dolor o molestias visuales, irritabilidad, dolor de cabeza, disminución de la visión, agotamiento físico, dolor o adormecimiento de las extremidades, dolor quemante en el estómago, dificultad respiratoria al subir escaleras, dolores articulares, disminución de la audición, dolor o molestias de oídos, mareos etc. Ibíd. p. 42. Ver en detalle todo el capitulo 3 del estudio.
Los conductores están expuestos al riesgo de atracos, especialmente en la noche y en barrios marginados o a amenazas de la guerrilla en partes de la ciudad de Cali.

\section{El transporte público colectivo desde el punto de vista de las empresas que prestan el servicio de buses:}

A pesar de ser los 'dueños' del negocio, tampoco es buena la situación para las empresas de transporte y los propietarios de los buses.

Incurren en múltiples sobre-costos que afectan la rentabilidad. Las empresas tienen costos elevados por las necesidades de reparaciones como consecuencias del maltrato de los buses por los conductores, que se estima en un 100\%; por los altos costos de seguros por los constantes accidentes, 15 estimados en un 100\%; el alto consumo de combustibles por el constante freno y aceleramiento del bus, que se estima en un $50 \%$.

\footnotetext{
${ }^{14}$ Como pasajero uno puede observar el manejo de los conductores, moviendo la palanca de cambios con fuerza, frenando y acelerando excesivamente, etc. El desgaste del motor y de otras partes del bus debe ser mucho más elevado que en un bus que se maneja con tranquilidad, que sólo para en los paraderos según un horario establecido donde hay una mínima presión y ningún interés por parte del conductor de acelerar la vuelta como es el caso de los sistemas organizados en ciudades europeas. Por falta de datos precisos se estima que los costos de mantenimiento aumentan en por lo menos $100 \%$ en comparación con un manejo 'sano' del bus.
}

15 En las condiciones laborales se ha mencionado que más que $30 \%$ de los conductores tuvieron en los pasados 6 meses un accidente de tránsito. Matar a un transeúnte puede causar riesgos financieros para el propietario o conductor de dimensiones incalculables si sus familiares exigen una indemnización. Se estima un sobre-costo de por lo menos $100 \%$ de las actuales primas de seguros de accidentes para el bus.

\footnotetext{
16 Se sabe que el consumo de combustibles aumenta significativamente cuando se acelera permanentemente. Si los buses paran por lo menos 5 o 6 veces como en un sistema organizado con horarios y paraderos, se estima el aumento del consumo en un $50 \%$.
} 
Los buses tienen una reducida vida útil por los maltratos de los conductores. ${ }^{17}$ Se estima estos sobre-costos en un 30\%. También los tiempos de

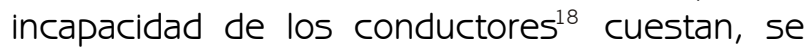
estima en cerca de un $5 \%$ más de lo normal.

Se necesitarían muchos más buses para prestar un buen servicio en Cali. El número de buses y recorridos requiere más inversión en control, administración, parqueo etc..$^{19}$ Se estima los sobre-costos en un $50 \%$.

La ocupación promedio de los buses es baja, los costos fijos de capital elevados?o Se calcula la

17 Acelerar constantemente es una carga alta para el motor, los frenos y otras partes mecánicas del bus (por ejemplo abrir y cerrar las puertas constantemente). Se estiman en $30 \%$ los sobrecostos.

18 Las condiciones laborales de los conductores conducen a enfermedades que pueden ocasionar la incapacidad de trabajar. Lo mismo pasa en casos de accidentes de tránsito u otros accidentes de trabajo. Se estima en $5 \%$ los costos adicionales.

19 Según un estudio de la Secretaria de Tránsito y Transporte Municipal, del año 2000, los buses en Cali ofrecen 1.667.880 lugares y transportan 1.282.201 pasajeros por día. El sistema transporta 1.35 pasajeros por $\mathrm{Km}$. en promedio, y hay una rotación de 2.11 personas por lugar. Las cifras muestran una baja ocupación de los buses, más aún, cuando se sabe que están llenos sólo en horas pico. Observaciones personales dan la impresión que la ocupación es en promedio muy baja, cuando en buena parte del día los buses pasan casi vacíos. Se puede estimar que hay una sobreoferta de cerca del $50 \%$ de buses en la ciudad, con las necesidades de control.

20 Una ciudad como Curitiba, Brasil, mueve diariamente cerca de 2 millones de personas con algo más de 1.500 buses (entre ellos buses articulados y biarticulados), en comparación con los cerca 5.000 buses en Cali que mueven por día cerca de 1.5 millones de pasajeros. Ver sobre Curitiba: Ramirez /Collazos/Alvarez. El favorecimiento y la reorganización del transporte público en Curitiba, Brasil. Trabajo de semestre, Facultad de Ingeniería, Universidad del Valle, agosto 1999, p. 9, Revista Trialog Mo 61. Towards the Sustainable City? Karlsruhe/Kiel2/1999. Se estima que en Cali se podría prestar un servicio integral con menos que la mitad de los buses que actualmente hay en la ciudad. ocupación promedio en cerca de $40 \%{ }^{21}$ incluyendo la alta ocupación en horas ${ }^{22}$ pico. Se estima que con la mitad de los buses se podría prestar un servicio eficiente en la ciudad.

Las empresas tienen conflictos: Con los conductores por las condiciones laborales, por el robo de una parte de los ingresos, ${ }^{23}$ por el maltrato de los buses, por accidentes. Con los afiliados porque éstos tienen dificultades para ganar suficiente para obtener utilidades. Con la Secretaría de Tránsito y el Municipio por las tarifas, 24 por las autorizaciones de nuevas rutas ${ }^{25} \mathrm{y}$ buses, por el abandono de partes de rutas etc.

Las empresas corren el riesgo de la disminución de la demanda, en situación de crisis económicaª.

21 MELO GARCíA, Ibíd.

22 El estudio de la Secretaría de Tránsito menciona como una de las causas de la reducción del número de pasajeros en los últimos diez años el incremento del número de motos en la ciudad que ha llegado a 70.000. Ibíd. p. 45.

23 Como pasajero se puede observar cuántas veces las personas suben al bus por la puerta atrás, no pocas veces provocado por el mismo conductor que para de tal manera que casi 'obliga' al pasajero a subir por atrás. Otras veces, como consecuencia de la crisis económica y con cifras en aumento, hay personas que piden poder pagar menos que el precio oficial para ser transportados. Estos pasajeros no aparecen en las estadísticas de los pasajeros transportados en la ciudad. Para el conductor contratado estos significan ingresos adicionales. Se estima en entre 5 y $10 \%$ de los viajes registrados.

${ }^{24}$ En las negociaciones entre las empresas y la Secretaría de Tránsito/el Municipio, sobre las tarifas, siempre aparecen los argumentos de las empresas que las tarifas no cubren los costos del servicio.

25 En septiembre de 2000 se desató una confrontación entre la Secretaria de Tránsito y algunas de las empresas transportadoras en Cali, por la autorización de busetas adicionales para una parte de las empresas que operan en Cali.

26 La demanda del transporte público colectivo en Cali ha disminuido en los años 1998 y 1999. Según datos de la Secretaría de Tránsito Municipal el sistema transporta en el año 2000 a cerca de 1.3 millones de 
Muchos de los usuarios del transporte público colectivo tratan de dejar el bus y comprarse una moto o un carro.

La ciudadanía critica a las empresas por el mal servicio, el comportamiento monopolístico, los altos precios del pasaje, la dominación o manipulación de las instituciones del estado y la supuesta influencia de negocios ilícitos.

Las empresas y los afiliados están constantemente expuestos a los riesgos de arrojar pérdidas en vez de ganancias, como consecuencia de los sobre-costos, del aumento de los precios de los combustibles, de los robos, de la reducción de la demanda etc. Como se puede ver en el estudio sobre las condiciones laborales de los conductores, cerca del $40 \%$ de ellos son propietarios o propietarios en asociación. Cerca del $70 \%$ de los propietarios logran ingresos de hasta tres salarios mínimos, poco más del 30\% logran ingresos de hasta 4 salarios mínimos, y cerca del $11 \%$ ingresos de más de 4 salarios mínimos? 28 Un factor de riesgo es que estos ingresos se logran porque los propietarios no realizan o no calculan adecuadamente los costos de un mantenimiento adecuado de sus buses.

\section{El Transporte Colectivo desde el punto de vista de la Ciudadanía, del Sector Empresarial y del Municipio:}

Aunque no están directamente involucrados, la ciudadanía, el sector empresarial y las instituciones y políticos del municipio están

pasajeros, 13.3 \% menos personas por día que en el año 1990. Se explica la disminución de la demanda, entre otros, por el aumento del número de carros y motos. Diagnóstico del Sistema de Transporte Público Colectivo en Cali, Secretaría de Tránsito y Transporte Municipal, Cali 2000. P. 43

27 Según informaciones recibidas en septiembre de 2000, una de las empresas había retirado casi todos los buses con motores de gasolina, porque el aumento fuerte del precio de este combustible hizo que su uso no fuera rentable, dada la baja demanda de pasajeros.

${ }^{28}$ Corporación Salud y Desarrollo / ISS Protección LaboralAdministradora de Riesgos Profesionales, El bienestar del conductor. afectados negativamente por la actual situación del transporte público colectivo, entre otros por los altos niveles de contaminación en la ciudad.

Los buses, busetas, etc. del transporte público colectivo contribuyen significativamente a la contaminación atmosférica y al ruido en la ciudad.

Los trancones a los cuales contribuyen los buses cuando se frenan mutuamente $y$ paran en cualquier momento, constituyen una pérdida de tiempo ${ }^{29}$ para los pasajeros, para los demás en el tráfico, para el transporte de bienes y constituyen un aumento de costos para la economía local. La larga duración de los viajes es tiempo que puede aprovecharse para estar en familia, con los amigos o para descansar.

La pérdida de tiempo en los viajes, el ruido, la contaminación, la agresividad en las relaciones entre diversos grupos en el tránsito tienen como una de las consecuencias el aumento del estrés. Este estrés se manifiesta en relaciones sociales más agresivas y violentas en el tránsito, y se transmite hasta al interior de las familias. Son más probables relaciones tensas en las empresas, y las peleas en casa cuando los pasajeros vuelven agotados después de un largo viaje en bus.

Los largos viajes, la contaminación y el ruido afectan la capacidad laboral de los empleados y obreros. Cuando las personas llegan cansadas y estresadas a la empresa es inevitable que su concentración, capacidad de dedicación e interés en el trabajo estén disminuidos, y que las relaciones de trabajo se tornen más complicadas.

\footnotetext{
$295 i$ se pudiera lograr un aumento de la velocidad promedio actual de los buses, de $20 \mathrm{~km}$. / h, a $30 \mathrm{~km}$. / h, el ahorro de tiempo sería impresionante.

30 Es difícil determinar el porcentaje en el cual se reduce la productividad de obreros y empleados, por ejemplo a través del aumento de errores por falta de concentración. Pero si se estima sólo en 2 o $3 \%$ en promedio, el valor económico en el Producto Interno Bruto local es importante, y constituye una disminución de la competitividad de las empresas.
} 
Para inversionistas y para personas de alta calificación es cada vez más un factor de importancia la situación ambiental y el 'civismo' de una ciudad: si el transporte está bien organizado y tranquilo, si el comportamiento de las personas es pacífico, etc., es más atractivo vivir y trabajar en una ciudad. El transporte urbano caótico, al contrario, disminuye el atractivo de la misma.

\section{La Causa Estructural de la Guerra del Centavo}

La mayoría de los problemas anteriormente descritos tienen una causa estructural en común: la organización del transporte público colectivo bajo condiciones de competencia desenfrenada que conducen a la llamada 'guerra del centavo'.

La 'guerra del centavo' tiene tres elementos estructurales:

1.La existencia de diferentes empresas de transporte $^{32}$ que compiten por las rutas más atractivas. Las más atractivas son las que más ingresos dan porque permiten transportar a más personas. La consecuencia es una sobreoferta de buses en sitios donde hay alta

${ }^{31} \mathrm{La}$ importancia relativa de este elemento de la competitividad de una ciudad o región es también difícil de calcular en términos económicos precisos. Pero claro es que mega-ciudades como Ciudad de México, Sao Paulo o Bogotá están afectadas por altos niveles de contaminación y el 'caos' que sufren sus habitantes a diario en su movilidad. Personas con altos niveles de calificación incluyen estos aspectos en la decisión sobre el traslado a un trabajo, e inversionistas los tienen en cuenta en su decisión sobre la ciudad en la cual quieren invertir.

${ }^{32}$ El sistema de transporte público colectivo de Cali está conformado por veintinueve (29) empresas de las cuales 15 prestan el servicio en buses, 3 en microbuses y 5 en camperos. Las empresas restantes seis (6), prestan el servicio en las diferentes clases de vehículos. Ver Estudio sobre el Sistema de Transporte Público Colectivo de Santiago de Cali, realizado por la Secretaria de Tránsito en el año 2000. P. 7/8. demanda, y la falta de servicio de transporte donde no hay o sólo hay pocos pasajeros.33

2. Los ingresos de las empresas y de los conductores dependen del número de pasajeros que transportan por despacho. Para garantizar utilidades las empresas tienen que buscar pasajeros a toda costa, y lo mismo vale para el conductor cuyos ingresos también dependen en buena parte del número de pasajeros.34 Por eso están obligados a realizar cualquier maniobra para recoger y dejar pasajeros en cualquier lugar, y a abandonar las rutas que no tienen pasajeros.5.

33 Este hecho es consecuencia de la lógica de la economía de mercado en la cual la empresa privada no tiene como función primordial la satisfacción de las necesidades de la población (en nuestro caso permitir la movilidad de todas las personas), sino la de producir utilidades. Una empresa que ofrece servicio de transporte en zonas donde sólo hay una demanda baja, que no cubre los costos y produce consecuentemente pérdidas, está condenada a desaparecer del mercado. La empresa tiene que buscar el máximo de la utilidad y en el transporte urbano actual éstas son las rutas con más demanda. En teoría, las empresas podrían prestar el servicio de transporte y generar pérdidas con unas rutas y ganancias mayores con otras rutas, con utilidades 'moderadas' en promedio. La realidad del servicio de transporte urbano muestra que las empresas no actúan así. En consecuencia, en la ciudad no hay servicio donde no hay demanda (=en zonas muy alejadas o en la noche, muy temprano o en días festivos cuando sólo podría haber pocos pasajeros).

34 Los conductores que no son propietarios de un bus reciben generalmente un ingresos básico y una suma fija por pasajero. Para controlarlos existen registradoras en las entradas del bus. Pero observaciones a diario muestran que los conductores permiten o provocan la subida de pasajeros por la puerta trasera para quedarse con el precio del pasaje. En otras palabras mejoran sus escasos ingresos robándole al propietario de bus o a la empresa.

35 MELO GARCÍA describe la 'guerra del centavo' así: "Los conductores deben entregar al propietario, entre $\$ 90.000$ diarios en el caso de los microbuses y $\$ 180.000$ pesos en los buses ejecutivos. Además, deben destinar entre $\$ 30.000$ y $\$ 50.000$ adicionales para tanquear el bus. Por eso cada conductor tiene que producir para cubrir costos y, además, su propio sueldo. "Todo lo que trabajamos hasta las 7:00 de la noche es para el patrón y para el carro. Por eso 
3. La subcontratación de afiliados y contratistas por parte de las empresas que tienen la licencia para el servicio en la ciudad. Estas empresas ganan más cuanto más afiliados tienen, y por cada despacho adicional, lo que provoca una sobreoferta de buses ${ }^{36}$ y la subsiguiente sobreinversión de capital.

Bajo las condiciones actuales de competencia desorganizada existe una lógica del comportamiento de todos los actores que empeora la situación en conjunto.

El pasajero por ejemplo sabe que el conductor está dispuesto a recogerlo y dejarlo en cualquier parte. Es lógico hacer parar el bus tan cerca de la casa como sea posible, 'ganando' unos minutos para no tener que caminar hasta un paradero más alejado. Pero lo mismo hacen muchos otros pasajeros en el transcurso del viaje. Cada uno 'gana' supuestamente un minuto, y todos juntos pierden mucho tiempo en el constante pare y acelere del bus.

El conductor por su lado tiene que recoger y dejar pasajeros en cada momento porque de ésto dependen sus ingresos y la existencia de la empresa. Si no lo hace se afecta negativamente a sí mismo y a las utilidades de la empresa o del propietario del bus. Por eso es lógico esperar para poder recoger más pasajeros en un sitio, o ir muy

tenemos que trabajar hasta las 10:00 y 11:00 p.m. para salvar lo nuestro." dijo Carlos Vargas, un conductor de microbús. "Aquí le toca guerrear a uno, no sólo con los de la misma empresa, sino con los de otras empresas para poder hacer algo, comentó el conductor Jorge Eliécer Zapata." Ibid. p. 2B

36 "La relación del parque automotor por empresas y su distribución ya sea activo, en mantenimiento o en reserva, de acuerdo con la modalidad del servicio, se tienen las siguientes conclusiones:

De los 4.868 vehículos registrados a la fecha del estudio, 2.609 (53.6\%) corresponden a vehículos tipo bus, 261 (5.4\%) a busetas, 1.383 (28.4\%) a microbuses y 615 (12.6) a camperos.

De las 29 empresas de transporte público inventariadas ninguna presenta parque automotor en reserva."

Ver Estudio, ibid. P. 8 rápido para estar siempre delante de otro bus de la misma empresa, como también esperar en la mitad de la calle a un pasajero, etc. Consecuentemente los viajes se vuelven mucho más lentos de lo normal, y aumenta el estrés para el conductor.

La empresa con licencia debe estar interesada en contratar más afiliados y subcontratistas, y de despachar más buses, porque estos son ingresos fijos. Mo importa que en el mismo momento la rentabilidad de los afiliados se disminuya constantemente.

El 'caos' actual es - en consecuencia - el resultado de la actuación aparentemente 'lógica' de todos los actores que se encuentran en una situación de competencia desorganizada. Cuando se aumenta la oferta de rutas y de buses, sin poder aumentar el número de pasajeros, y las empresas dependen de los ingresos por número de pasajeros, el resultado es adverso a lo que se está esperando. Más oferta no mejora el servicio para los pasajeros y ciudadanos de la ciudad, sino que empeora finalmente la situación para todos: pasajeros, empresas, conductores, el medio ambiente natural y la ciudadanía sufren por igual.

\section{EXIGENCIAS Y CRITERIOS QUE TIENE QUE CUMPLIR UN SISTEMA DE TRANSPORTE PÚBLICO COLECTIVO}

Antes de proponer una solución es necesario definir las exigencias y criterios que debería cumplir un buen sistema del transporte público colectivo.

\section{La organización de las rutas y paraderos:}

El sistema de transporte debe cubrir toda la ciudad con una buena oferta, debe haber sólo una ruta por calle para evitar que los buses se frenen mutuamente, las rutas y frecuencias deben reflejar la demanda. Los buses deben recoger y dejar pasajeros exclusivamente en los paraderos para evitar la pérdida de tiempo en las constantes 
paradas en cada esquina. Debe haber una integración con el transporte intermunicipal en la región metropolitana de la ciudad.

\section{Frecuencias, horario y conexiones}

El sistema debe ser calculable y confiable para el pasajero, debe haber un horario fijo para cada paradero o la definición de frecuencias. El pasajero debe poder calcular cuánto tiempo necesita para llegar a cualquier punto de la ciudad. El sistema debe cumplir frecuencias mínimas para ciertas horas del día, debe haber un servicio nocturno. Los puntos de conexiones entre rutas deben permitir el traslado dentro de pocos minutos de espera, y la velocidad del sistema debe permitir cruzar una ciudad como Cali (con una distancia de cerca de $16 \mathrm{~km}$. de un lado al otro) en 45 minutos desde cualquier punto de partida en el perímetro urbano37

\section{Tipo y calidad de los buses}

Debe haber rutas exprés de alta capacidad de pasajeros (pueden ser buses articulados) y rutas alimentarias que lleven los pasajeros desde los barrios de vivienda a las rutas exprés. Los buses deben tener un interior cómodo y agradable, estar bien iluminados en la noche, con sillas cómodas y ventilación buena bajo las condiciones climáticas de Cali. Los buses no deben tener gradas para así permitir la subida con sillas de rueda y de otros pasajeros con limitaciones físicas. Para acelerar la subida y bajada de pasajeros debe haber puertas amplias para el movimiento de más que una persona al mismo momento.

\section{Estructura de tarifas, tipo de tiquete y pago, precios:}

La tarifa debe permitir la movilidad de toda la

\footnotetext{
${ }^{37}$ La velocidad promedio del sistema actual es de 20 $\mathrm{Km}$. / h. Con una velocidad de $30 \mathrm{~km}$. / h se podría lograr este objetivo.
}

población de la ciudad sin excluir a nadie por altos costos. Se pueden pensar dos alternativas de tarifas:

$>$ Una tarifa única que permite moverse en toda la ciudad y cambiar entre todas las rutas con un solo tiquete. La tarifa única desfavorece a las personas que sólo viajan cortas distancias y a los hogares con bajos ingresos con más de una persona que quiera movilizarse.

> Tarifas diferenciadas por distancias recorridas: para cruzar toda la ciudad se paga más que para moverse dentro del barrio o unas pocas paradas. Así se puede bajar los costos del transporte y dar opción de movilidad a más personas, en especial en los estratos bajos.

\section{Con Respecto a los Paraderos:}

Los paraderos deben proteger del sol, del viento y de la lluvia, permitir la entrada de personas con cualquier limitación física (discapacitados), deben ofrecer en el futuro la venta anticipada de tiquetes a través de dispensadores automáticos o por cajeros. Puede haber una oferta de servicios y ventas por parte del sector informal, pero deben ser limpios, agradables, con opción para sentarse y la iluminación adecuada en la noche.

\section{Para los conductores:}

Los conductores tienen el derecho a condiciones laborales sanas, con un máximo de 8 horas laborales por día, y con ingresos que le permiten a ellos y a sus familias una vida digna.

\section{Para las empresas y propietarios de buses:}

A las empresas y los propietarios de los buses, y a todos los que tienen inversión en el sector, el transporte público colectivo debe garantizar una rentabilidad razonable comparable con otros sectores económicos.

\section{Para el medio ambiente natural, la ciudadanía y los sectores económicos:}

El transporte público colectivo debe contaminar el aire y producir ruido lo mínimo posible, debe 
fomentar la convivencia pacífica y la equidad entre los ciudadanos y favorecer la productividad y competitividad de los sectores económicos.

\section{Argumentos en contra del tren ligero para Cali:}

Entre las soluciones para el transporte público colectivo en grandes ciudades se discute el tren ligero o metro.

Teniendo en cuenta las exigencias y criterios presentados arriba, que debería cumplir el sistema de transporte público colectivo, existen muchos argumentos en su contra, por lo menos para el caso de Cali:

$>$ La zona de influencia directa del tren ligero o de un metro siempre sólo cubrirá una pequeña parte de la superficie de la ciudad: en Cali cerca del $10 \%$ de la superficie metropolitana. ${ }^{39}$

$>$ Va a mover consecuentemente sólo una

38 Mo hay espacio para una evaluación detallada del nuevo sistema Transmilenio que se está desarrollando en Bogotá. El sistema consiste en grandes buses articulados que circulan en carriles propios y aislados en las grandes troncales de la ciudad. El sistema exige la construcción de estaciones elevadas para permitir la entrada de los pasajeros a nivel de las puertas del bus. Además se tuvieron que adecuar los carriles para separarlas del transporte vehicular particular lo que requiere inversiones elevadas. El sistema pretende evitar los efectos de la 'guerra del centavo' porque no hay otros buses en las calles en las cuales hay Transmilenio. El sistema es mucho más barato que un metro o un tren ligero. Hay conflictos con los propietarios de buses que se sienten excluidos y desplazados por Transmilenio. Mo se ha organizado la integración con las otras rutas de buses. Mo se sabe qué va a pasar con los propietarios de buses y los conductores que van a ser innecesarios. El precio del sistema es elevado. Se podría formular dudas de si es necesario un costoso sistema satelital para controlar velocidades y el cumplimiento de horarios.

39 La zona de influencia se define con 500 metros a ambos lados de los carriles. Cuando es más, la distancia y el tiempo para caminar hasta la estación del tren es tan grande que no es atractivo usar el tren ligero o metro. pequeña parte de la demanda: en Cali cerca del $10 \%$ de las personas. ${ }^{40}$

$>$ Consecuencia: la calidad del transporte público colectivo dependerá del sistema de buses. Siempre los buses tendrán que mover la gran mayoría de las personas.

Muy altos costos: en Cali se estiman entre 600 y más de 1000 millones de dólares, para un tren ligero de cerca de $18 \mathrm{Km}$. de rutas.

- Largas distancias entre estaciones / paraderos. Lo que uno gana con la rapidez del tren, lo pierde caminando largas distancias o subiendo y bajando las gradas de la estación subterránea. ${ }^{41}$

$>$ Las inversiones quedan principalmente en el exterior. Los trenes se producen en países extranjeros.

- Es una carga financiera alta para el municipio y la nación. Para poder financiar la inversión, la ciudad y la nación deben endeudarse con la banca extranjera, lo que significa una carga alta de intereses en el futuro.42

$>$ Los ingresos difícilmente cubren los gastos de funcionamiento y mantenimiento . $^{43}$

$>$ La estructura física de la plataforma urbana tiene los efectos de un 'muro de Berlin'. O la estructura física del metro destruye la imagen del centro de una ciudad como Medellín, o constituye una barrera prácticamente insuperable entre los barrios. ${ }^{44}$

40 En Medellín el metro mueve sólo $9 \%$ de la demanda de la ciudad, según las palabras del propio gerente, Álvaro Bernal. UMIDAD INVESTIGATIVA. Empeñado el metro de Medellín. El Tiempo. Septiembre 12 de 1999. p. 8 A

41 En Cali está previsto una estación por cada kilómetro de ruta. Es una consecuencia inevitable de los altos costos de su construcción.

42 El metro de Medellín no ha podido pagar sus deudas con la Mación, los ingresos por operación ni cubren los pagos de funcionamiento, en ninguno de los años desde 1996. UNIDAD INVESTIGATIVA. EI Tiempo, ibid.

43 UMIDAD INVESTIGATIVA. IDId.

44 La construcción prevista de unos pocos puentes peatonales que cruzan los carriles del tren ligero, no cambian esta situación. 
/ Hay alternativas mejores, mucho más eficientes y económicas.

\section{LA PROPUESTA PARA EL TRANSPORTE PÚBLICO COLECTIVO EN CALI ${ }^{45}$}

Seguidamente se exponen los principales elementos de la solución para el transporte público colectivo en Cali. Las propuestas son principalmente organizacionales, con complementos tecnológicos4.6

\section{Crear una empresa única de los transportadores en Cali:}

Para eliminar la nefasta 'guerra del centavo' se puede crear una empresa única para el transporte público colectivo en la ciudad. De esta empresa formarán parte y serán propietarias todas las empresas, todos los propietarios de los buses, todos los conductores que actualmente prestan el servicio o tienen inversión en el sector, con el valor de su inversión (como 'accionistas') o como conductores. De lo que actualmente hay no se pierde nada. Mo se excluirá ninguno de los actualmente involucrados, y tampoco nadie perderá su puesto de trabajo. En la Junta Directiva de la empresa unificada el municipio debe tener un peso de decisión relevante.

Una función importante de la empresa unificada

\footnotetext{
${ }^{45}$ El transporte público colectivo es un importante elemento en el transporte urbano. Otro elemento será crear condiciones seguras para ir a pie o ir en bicicleta en toda la ciudad. Ver MOLLER, Rolf. Propuesta para la creación de condiciones seguras para la movilidad de peatones y ciclistas en Santiago de Cali. Junio de 2000. Presentada al Consejo Municipal de Cali para la discusión del POT.

46 Para la propuesta se retomaron las experiencias en Curitiba, Brasil; en Quito, Ecuador con su trolebús; en ciudades europeas como Hamburgo, que tiene el tamaño de Cali, Frankfurt y Paris. Varias de las ideas presentadas también se encuentran en cierta forma en las propuestas que fueron presentadas en Cali a comienzos de los años '90.
}

será organizar una oferta estructurada y adecuada para la demanda en la ciudad. La empresa define las rutas, las frecuencias y horarios, el reemplazo de dos o tres buses viejos por uno nuevo y menos contaminante ${ }^{48}$ y otras inversiones. La empresa será controlada por el municipio, con participación directa de representantes de los pasajeros, y con voz y voto de una representación de los conductores contratados en la Junta Directiva.

La empresa unificada recibe los ingresos por parte de los conductores y tiene la función de la distribución según los mecanismos descritos abajo.

\section{Eliminar la 'guerra del centavo': pagar por kilómetros recorridos, y no por número de pasajeros}

Como se ha mostrado arriba, un elemento estructural de la 'guerra del centavo' son los ingresos y utilidades según el número de pasajeros. Consecuentemente, en la empresa unificada las empresas y propietarios de los buses y los conductores no reciben una remuneración por el número de pasajeros transportados en un recorrido, sino que se paga por kilómetro recorrido y según el tipo de bus (buses grandes, buses articulados, busetas, microbuses, camperos). La empresa define, con base en estudios de costos, el valor por kilómetro recorrido de cada tipo de bus. Los conductores reciben un salario fijo para un servicio de máximo 8 horas de trabajo diario.9 El salario debe permitir una vida digna para ellos y sus familias.

\footnotetext{
47 La empresa define la reducción del número de buses a un nivel de 'capital' razonable, y en qué tiempo se realizan los cambios. Para acelerar el cambio hacia buses modernos puede ser muy útil la ayuda de la Mación que estaba prevista para la construcción del tren ligero.

480 la reconversión tecnológica a gas natural.

495e podría pensar en un salario cerca a 4 salarios mínimos.
} 


\section{Organizar una estructura integrada de rutas:}

En la ciudad se organiza una estructura integrada de rutas: rutas exprés para la alta demanda, y rutas alimentadoras desde los barrios y sitios más alejados de la ciudad. Las rutas van a cubrir toda la ciudad, con una distancia máxima de 500 metros desde la casa hasta el paradero más cercano.

Pero no será una buena solución con muy pocas rutas exprés como se ha pensado el tren ligero o Transmilenio en una versión para Cali, donde sólo están previstas dos o tres rutas principales, una de norte a sur y otra desde Aguablanca hasta el centro. Por supuesto que los vagones del tren ligero y los buses de Transmilenio en sí son agradables. Pero: primero, la concentración de un gran número de pasajeros en muy pocas rutas exige sistemas de transporte masivo, como vagones del tren ligero o buses articulados que requieren una infraestructura con 4 carriles exclusivos, en el caso del bus articulado. La infraestructura será muy costosa y como 'un muro de Berlin', partiendo la ciudad en partes desconectadas. Segundo, el transporte será lento desde el punto de vista del pasajero. El tren ligero o los buses articulados serán por sí solos muy rápidos. Pero requieren cambiar dos veces de bus.

La gran mayoría de los pasajeros estará obligada a tomar primero un bus alimentador desde el barrio para llevarlo a la estación del tren ligero o Transmilenio. Tiene que bajar del bus, cruzar la calle para entrar a la estación, subir al tren ligero o al bus articulado, ir a una estación cerca del destino, para tomar otra vez un bus que lo llevará finalmente a su destino. Un viaje de Aguablanca al centro de la ciudad - de máximo 8 kilómetros puede durar hasta 30 minutos y más. De éstos sólo 10 serán en el tren ligero o el bus articulado.

Más rápido será una estructura de múltiples rutas no muy distantes, paralelas, con conexiones cada 5 ó 6 cuadras. Para estas rutas se puede, sin problemas, usar buses tamaño 'ejecutivo', para cerca de 60 pasajeros.
En los paraderos se señalarán los horarios fijos y / o las frecuencias, los paraderos y destinos por los cuales pasa la ruta, y la estructura completa de rutas en la ciudad para facilitar la orientación de los pasajeros.

Si excepcionalmente la demanda lo justifica, las rutas exprés podrían ser servidas con buses articulados. Las rutas alimentadoras y el servicio de noche se presta en busetas o microbuses, según la demanda.

Se asegura una conexión en los puntos de conexión dentro de un tiempo definido, por ejemplo 5 minutos en horas pico. Se asegura una conexión rápida también en días festivos y en la noche, a través de un horario establecido.

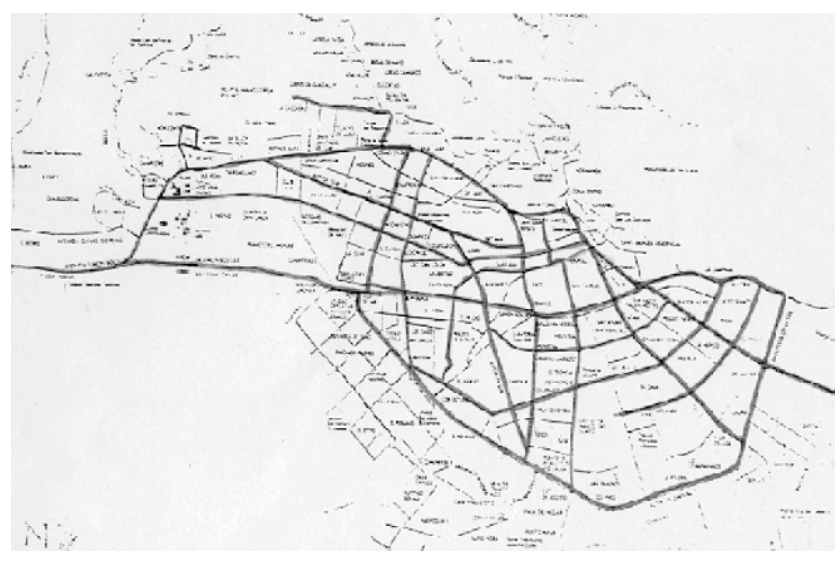

El mapa muestra cómo se podría pensar la organización integrada de rutas en la ciudad. La densidad de las rutas permite el uso de buses tipo 'ejecutivo', sólo si la demanda lo exige se requiere buses articulados. De los barrios salen las rutas alimentadoras para llevar al pasajero a estas rutas con altas frecuencias. Una opción es la tarifa única, Con un solo tiquete el pasajero puede moverse en toda la ciudad. Otra opción es la tarifa diferenciada: cuanto más largo el viaje, cuanto más cuesta el tiquete.

\section{Realizar la integración del transporte público en la ciudad con el transporte intermunicipal en la región metropolitana:}

Se propone la integración de las rutas que conectan los municipios con la región metropolitana de la ciudad, en el sistema del transporte público dentro de la misma. Los buses intermunicipales cruzan toda la ciudad, por 
ejemplo Jamundí - Yumbo. En el casco urbano de Cali se vuelven una oferta de transporte muy rápida y cómoda tipo clase ejecutiva de un avión. Los buses sólo paran en puntos estratégicos de conexiones importantes y a grandes distancias (por ejemplo: sólo cada tres kilómetros). Por ser rápidas y cómodas, estas rutas van a atraer a pasajeros que hoy en día utilizan el carro.

\section{Utilizar la red vial ya existente como infraestructura (exclusiva) para los buses:}

Para las rutas reorganizadas de los buses no es necesario construir una plataforma urbana, con altos costos y el carácter de un 'muro de Berlin' 5 . Se utilizan las calles y carriles de la red vial ya existente. Donde existe el riesgo de congestionamiento por el alto flujo vehicular particular, se reserva uno de los carriles exclusivamente para los buses del transporte público colectivo, separándolos físicamente a través de bolardos, varillas de acero u otro tipo de separadores duros de los carros particulares.' Como ya no existirá competencia entre varios buses en la misma calle, un solo carril es

50 En este sentido la propuesta para Cali es diferente del sistema Transmilenio de Bogotá, que requiere la construcción de estaciones con una plataforma elevada. Se piensa en buses cuya entrada se encuentra cerca de la altura del anden, sin gradas. Estos buses por supuesto no pueden ser construidos como los de hoy sobre la estructura de un camión. Se puede inclusive pensar en buses con un mecanismo hidráulico que bajan su lado en el paradero hasta el nivel del anden. Este tipo de bus conoce el autor de su ciudad natal en Alemania, Gütersloh.

51 Así se hace por ejemplo en el centro histórico de Quito. Con carriles exclusivos para buses se redistribuye el espacio que ocupan hoy en día miles de buses que se bloquean mutuamente. Mo está previsto devolver este espacio ahorrado a los carros. Al contrario, carriles exclusivos para buses devuelven algo de equidad al sistema de transporte público colectivo y a la gran mayoría de los ciudadanos. Mo se debe olvidar que algo como $10 \%$ de la población con carros ocupan actualmente cerca del $80 \%$ de la infraestructura vial, un bien público. suficiente para su flujo continuo hasta con frecuencias muy altas. Así se asegura el flujo sin interrupción de los buses, con una velocidad que permite cruzar la ciudad en 45 minutos.

\section{Tomar medidas adicionales para dar prioridad al transporte público colectivo:}

Son necesarias otras medidas para crear al transporte público colectivo condiciones de igualdad en comparación con los vehículos particulares y para hacer el sistema atractivo también para las personas que hoy en día se mueven en carro. Hay que recordar que en Cali 5.000 buses mueven actualmente cerca del $80 \%$ de los pasajeros, mientras 250.000 carros mueven apenas poco más del 10\%. Las medidas para favorecer y acelerar el transporte en buses pueden ser, entre otras, las siguientes:

> Los ya mencionados carriles exclusivos, con separadores físicos, en calles donde puede haber trancones.

> Prioridad en semáforos: el conductor puede darle una señal electrónica al semáforo para que éste le de luz verde (ola verde para buses).

> Paraderos que permiten la subida al bus al mismo nivel de la puerta de entrada.

> Peatonalizar el centro de la ciudad, con el permiso de entrada sólo para buses del transporte público colectivo.

> Sistemas de prepago de tiquete, a través de cajeros en los paraderos, de dispensadores automáticos, de tiquetes prepago semanal o mensualmente.

> Multas severas para los propietarios de vehículos particulares que de una u otra manera bloqueen el transporte público colectivo en los semáforos o en los carriles exclusivos para buses.

\section{Ventajas de esta solución para crear un sistema eficiente, agradable y rápido de transporte público colectivo: ${ }^{52}$}

La propuesta presentada anteriormente tiene una serie de ventajas que enseguida vamos a 
presentar en detalle.

\section{Desde el punto de vista del servicio:}

La calidad del servicio aumentará significativamente. El pasajero sabrá a qué hora tiene que salir de un punto de la ciudad para llegar a la hora deseada al sitio de destino. El sistema cubrirá toda la ciudad, no hay exclusión de nadie, ni por pobre, ni por una limitación física. El tiquete de viaje puede costar menos que actualmente, especialmente para distancias cortas, permitiendo a más personas la movilidad deseada en la ciudad. ${ }^{53}$ Se reduce el tiempo para cruzar la ciudad a la mitad. Habrá un servicio nocturno 54 Habrá un servicio de lujo y alta velocidad suficientemente atractivo para reemplazar el vehículo particular.

La reorganización e integración de las rutas se puede realizar prácticamente desde ya, y la oferta sería completa en muy corto tiempo.

\section{Desde el punto de vista del medio ambiente y de la salud de los ciudadanos:}

Los buses se manejarán tranquilamente, sólo pararán en los paraderos. Circularán menos de la mitad de los buses actuales, prestando un

\footnotetext{
52 En la evaluación de la propuesta se aplican criterios de desarrollo sostenible, un concepto que incluye criterios sociales, económicos, ambientales y culturales. Para el concepto del desarrollo sostenible existen todavía una gran variedad de interpretaciones. Un buen resumen presenta Wolfgang Sachs. La anatomía política del desarrollo sostenible, y los otros artículos en el libro de Ecofondo / Cerec (editores). La gallina de los huevos de oro: Debate sobre el concepto de desarrollo sostenible. Bogotá 1996

53 Elementos del desarrollo sostenible son la creación de condiciones de equidad social y de disminución de la pobreza.

54 Hay que organizar en alguna manera una protección contra los atracos.

55 Bajo la suposición de que se logra un acuerdo inmediato entre todas las empresas de transportadores urbanos y de todos los propietarios de buses, con el municipio.
}

mejor servicio. Así disminuye significativamente la contaminación del aire y el ruido:56 El viaje se torna mucho más tranquilo, y se eliminan los riesgos de accidentes dentro y fuera del bus, que son un efecto de la 'guerra del centavo'.

\section{Desde el punto de vista de las empresas de transportadores y de los propietarios de buses:}

Todos los que entren en la empresa unificada van a tener un margen de utilidad razonable para su inversión. Se hará la transformación a un nivel razonable de capital (en forma de buses, infraestructura, talleres etc.), encontrando el equilibrio entre demanda y oferta. Creando una empresa única para todas las empresas y propietarios actuales, se evitan los conflictos que podrían paralizar el transporte en la ciudad.

\section{Desde el punto de vista de los conductores:}

Minguno de los actuales conductores va a perder su trabajo, al contrario, se mejorarán significativamente sus condiciones laborales. En vez de 14 horas diarias van a trabajar sólo entre 6 y 8 horas por día. Cada conductor va a trabajar por turnos. Se necesitarán cajeros dentro de los buses grandes, en los paraderos, más controladores para que los pasajeros compren su tiquete de viaje, etc. Los ingresos estarán garantizados independientemente del número de los pasajeros a un nivel que permita a ellos y sus familias una vida digna 58

\footnotetext{
${ }^{56}$ El elemento ambiental del desarrollo sostenible.

57 Pocos días antes de empezar la primera ruta de Transmilenio en Bogotá en diciembre de 2000 hubo la amenaza de un paro de los transportadores de buses que se sienten amenazados por el nuevo sistema de transporte público colectivo.

58 Hay que calcular el costo del 'recurso humano' para el sistema de transporte público colectivo, partiendo de los actuales niveles de ingresos, cerca de 3 ó 4 salarios mínimos. Hay que evitar privilegios a costa de la población.
} 


\section{Desde el punto de vista económico del Municipio y de la Mación:}

La reforma del sistema de transporte público colectivo empieza con los buses que ya están, y requiere poca inversión pública en infraestructura (los separadores en las partes de las calles en las cuales el bus tendrá su propio carril). mejoramiento del sistema - cambio de buses viejos por nuevos más cómodos y menos contaminantes - se hace, por un lado, con los ingresos de la empresa unificada; por otro lado, puede haber ayuda del municipio y la contribución de la Mación, pero con sumas mucho más bajas que las que se gastaron para el metro de Medellín, estaban dispuestos a gastar para el tren ligero de Cali y se van a gastar en el sistema Transmilenio en Bogotá. Mi el municipio, ni la Mación tienen que endeudarse con la banca extranjera para la creación de un buen sistema de transporte público colectivo en Cali. ${ }^{59}$

\section{Desde el punto de vista de la ciudadanía y de la paz social:}

La ciudad se vuelve mucho más tranquila, disminuye el estrés. Habrá más equidad social con más opciones de movilidad para los menos favorecidos en la ciudad. Los que usan el transporte público colectivo se sentirán tan respetados como los propietarios de carros hoy en día. Disminuyen muchos de los conflictos en el tránsito y baja la violencia.

\section{Desde el punto de vista de las empresas privadas y de la economía local:}

Los obreros y empleados van a llegar al trabajo mucho más tranquilos, menos cansados y estresados, lo que aumentaría la productividad en sus labores y disminuye conflictos laborales. Se perderá mucho menos tiempo en todo tipo de

59 Evitar un endeudamiento excesivo contribuye a la sostenibilidad económica y financiera de una inversión pública. Se libera plata para la inversión social en el municipio, en educación, vivienda social, cultura etc. viajes en la ciudad, lo que disminuye costos y aumenta la competitividad de sus empresas. La ciudad se torna más atractiva para la inversión (extranjera) por su tranquilidad, organización, la eficiencia en su sistema de transporte y el comportamiento cívico de la gente. ${ }^{60}$

\footnotetext{
${ }^{60}$ Cuando se trata de resolver problemas o conflictos, en muchos casos es difícil asegurar que todos salgan contentos con la solución y el compromiso encontrados. En la solución de problemas es deseable evitar situación de perder - perder o ganar - perder (todos pierden o uno gana, y el otro pierde). La propuesta presentada aquí para reformar el transporte público colectivo en Cali, crea la situación óptima de ganar - ganar: en la solución no pierde nadie, todos ganan.
} 